\title{
A TEORIA DO DÉFICIT DE AUTOCUIDADO DE OREM APLICADA EM HIPERTENSAS
}

Nágela Valadão Cade ${ }^{1}$

Cade NV. A teoria do déficit de autocuidado de OREM aplicada em hipertensas. Rev Latino-am Enfermagem 2001 maio; 9(3):43-50.

Estudo realizado com o objetivo de avaliar a Teoria do Deficit de Autocuidado de Orem (TDAC) em mulheres portadoras de hipertensão arterial. Foram entrevistadas quatro mulheres hipertensas em tratamento ambulatorial mediante formulário de entrevista aberta e fechada sobre os fatores condicionantes e os requisitos para o autocuidado. Os dados foram analisados segundo o referencial da TDAC. A partir dos requisitos de autocuidado que envolvem o hipertenso, foi identificada a existência ou não de adequação entre a capacidade e a demanda de autocuidado, e os facilitadores e dificultadores desta adequação. Conclui-se que a TDAC possibilitou identificar aspectos importantes do hipertenso para serem trabalhado pelo enfermeiro.

UNITERMOS: autocuidado, enfermagem, hipertensão

\section{OREM'S SELF-CARE DEFICIT THEORY APPLIED TO HYPERTENSIVE PEOPLE}

A study carried out with the objective of evaluating Orem's nursing self-care theory (TDAC) in women with hypertension. Four hypertensive women in an outpatient clinic were interviewed using a form with open and mulitple-choice questions on conditioning factors and self-care requirements. The data were analyzed according to the TDAC theoretical framework. Based on the self-care requirements for hypertensive people, the existence or non-existence of adjustment between self-care capacity and self-care need as well as the inhibitors and enhancers of such adjustment were identified. It was concluded that TDAC allowed to identify important aspects related to hypertensive people which must be dealth with by nurses.

KEY WORDS: self-care, Nursing, hypertension

\section{LA TEORÍA DEL DEFICIT DE AUTOCUIDADO DE OREM APLICADA A HIPERTENSAS}

Estudio realizado con el objetivo de evaluar la Teoría del Déficit de Autocuidado de Orem (TDAC) en mujeres con hipertensión arterial. Fueron entrevistadas cuatro mujeres hipertensas en tratamiento ambulatorio mediante un formulario de entrevista con preguntas abiertas y cerradas sobre los factores condicionantes y los requisitos para el autocuidado. Los datos fueron analizados según el referencial de la TDAC. A partir de los requisitos de autocuidado que envuelven el hipertenso, fue identificada tanto la adecuación entre la capacidad y la demanda de autocuidado como la ausencia de éstas, así como también las condiciones que favorecen y dificultan ésta adecuación. Se concluye que la TDAC posibilitó detectar aspectos importantes de la persona con hipertensión para ser trabajados por el enfermero.

TÉRMINOS CLAVES: autocuidado, enfermería, hipertensión

${ }^{1}$ Docente do Departamento de Enfermagem da Universidade Federal do Espírito Santo. Doutoranda da Escola de Enfermagem da Universidade de São Paulo.E-mail:nagela@npd.ufes.br 
INTRODUÇÃOO

A hipertensão arterial é uma doença crônicodegenerativa do sistema cardiovascular e o seu controle tem constituído um desafio para os profissionais de saúde, pois seu tratamento envolve a participação ativa dos hipertensos no sentido de modificar alguns comportamentos prejudiciais à sua própria saúde e assimilar outros que beneficiem sua condição clínica.

A hipertensão arterial essencial ou primária compreende de 90 a 95\% dos casos desta afecção e pode ser controlada desde que os pacientes envolvam-se em ações tais como uso da medicação anti-hipertensiva de forma regular, controle do peso, ingestão de dieta hipossódica com baixo teor de gordura saturada e colesterol e realização de atividade física, pois estas colaboram com a redução da pressão arterial em alguns pacientes e minimizam as complicações em órgãos-alvo.

0 modelo biomédico, que tem direcionado as ações de enfermagem hoje, prioriza a "doença observada" e detectada através das alterações na estrutura anatomoquímica do organismo, propondo uma terapêutica impositiva. Ocorre, no entanto, nos casos de doença crônica, as possibilidades de sucesso do tratamento estarem em sintonia com a "doença percebida", pois as ações de saúde serão realizadas pelos pacientes de acordo com a percepção, vontade, possibilidades e modificações que a doença e as formas de tratamento impõem às suas vidas ${ }^{(1)}$.

Um dos modelos que pode direcionar as ações assistenciais do enfermeiro e responder as necessidades do portador de doença crônica advém da Teoria do Déficit de Autocuidado, de Dorothea E. Orem. A autora considera a educação para 0 autocuidado* um processo dinâmico que depende da vontade do cliente e da percepção dele sobre sua condição clínica ${ }^{(2)}$.

Nela, os pacientes julgam se a ação de autocuidado é benéfica para eles, e esse julgamento ocorre de acordo com as orientações internas e/ou externas, que, por sua vez, são moldadas pela cultura em que os indivíduos vivem ${ }^{(3)}$.

0 postulado principal da Teoria do Déficit de Autocuidado (TDAC) é a incapacidade da pessoa em cuidar dela própria para atingir saúde e/ou bem-estar, e esse déficit ocorre quando há um desequilíbrio entre a capacidade para o autocuidado ${ }^{(4) * *}$ e a demanda terapêutica de autocuidado***.
0 problema de pesquisa constituiu-se então, a partir dos seguintes questionamentos: a) até que ponto o levantamento de dados, tomando como base os requisitos de autocuidado necessários a pessoa hipertensa, pode nos conduzir à demanda terapêutica e ao déficit de autocuidado para esta clientela? b) a TDAC aborda pressupostos teóricos que permitem, na prática do enfermeiro, levantar questões importantes que possam ser trabalhadas com 0 portador de hipertensão arterial?

A partir dessas colocações, pretende-se, com este estudo, aplicar a teoria do autocuidado com pacientes portadores de hipertensão arterial essencial. Os objetivos a que nos propomos constituem:

a) identificar os requisitos de autocuidado que se relacionam com 0 adulto portador de hipertensão;

b) detectar os fatores facilitadores ou dificultadores para a realização do autocuidado;

c) identificar o déficit de autocuidado na população estudada.

\section{METODOLOGIA}

Este é um estudo exploratório, descritivo, com análise qualitativa dos dados.

\section{Sujeitos}

Foram entrevistadas quatro pacientes hipertensas em tratamento no ambulatório da Liga de Hipertensão Arterial do Hospital das Clínicas - Faculdade de Medicina da Universidade de São Paulo, cidade de São Paulo.

Os critérios para a seleção da amostra foram: a) adulto, com idade entre 40 e 75 anos; b) sexo feminino; c) concordar em participar do estudo.

Optou-se por trabalhar com mulheres devido a possibilidade de fazerem uso de contraceptivos hormonais, sendo este um requisito de autocuidado importante no hipertenso. A coleta de dados foi interrompida após quatro entrevistas, quando os dados passaram a se repetir, e por ser este um estudo qualitativo.

\section{Instrumento de coleta de dados}

0 instrumento constituiu-se num formulário de entrevista

\footnotetext{
* Autocuidado "é a prática de atividades que o indivíduo inicia e realiza em prol de si próprio para manter a vida, a saúde e o bem-estar"(2)

** Capacidade para autocuidado é a habilidade do indivíduo para enganar-se no autocuidado ${ }^{(4)}$

*** Demanda terapêutica de autocuidado são os fatores que devem ser trabalhados, controlados e modificados no indivíduo por afetarem o funcionamento do organismo e seu desenvolvimento humano ${ }^{(2)}$
} 
com questões abertas e fechadas contemplando os seguintes aspectos: a) identificação; b) requisitos de autocuidado que compreendemos estarem associados ao portador adulto de hipertensão. São eles: requisitos universais (alimentação, exercícios e atividade física, interação social e promoção do desenvolvimento humano em grupos sociais lazer e recreação, prevenção de riscos, manutenção da saúde); requisitos relacionados ao desenvolvimento humano (uso de anticonceptivo hormonal), e requisitos relacionados aos desvios de saúde (percepção, expectativa e conhecimento sobre a doença e sobre a medicação anti-hipertensiva - dose, efeito colateral, dificuldades apresentadas e aquisição).

A incapacidade para desenvolver o autocuidado pode advir de alguns fatores externos como doença ou a morte de algum parente, ou internos, como idade, que afetam tanto a habilidade da pessoa engajar no autocuidado, como o tipo e a quantidade de autocuidado requerido. Esses determinantes externos e internos ao indivíduo foram denominados "fatores condicionantes" ${ }^{\text {(2) }} \mathrm{e}$ achamos pertinente incluí-los na coleta de dados. São eles: idade, estado de desenvolvimento, orientação sociocultural, estado de saúde, sistema familiar, padrão de vida, recursos usados para adequação (rede de suporte social).

\section{Operacionalização da coleta de dados}

Os dados foram colhidos e registrados no formulário pela pesquisadora, quando a paciente compareceu à Liga de Hipertensão Arterial para a consulta médica, na primeira semana de junho de 1998. As pacientes que satisfaziam os critérios de seleção averiguados previamente nos prontuários eram entrevistadas no local, após explicação do motivo da pesquisa e do aceite por escrito da mesma.

\section{RESULTADOS E DISCUSSÃO}

\section{Caracterização pessoal e familiar dos sujeitos}

Maria* tem 73 anos, casada, sem filhos, vive em casa própria com dois sobrinhos adultos órfãos, sendo um deles portador de doença mental. 0 marido é aposentado, com renda mensal de três salários mínimos e recebem ajuda financeira de um dos sobrinhos. Realiza todas as atividades domésticas sozinha, e apesar de não ter freqüentado a escola, lê e escreve bem, fazendo da leitura um passatempo.

Joana tem 58 anos, é viúva e mora com um irmão adulto portador de doença mental e a única filha casada, quatro netas e o genro. Há menos de um mês reformou a casa, dividindo-a ao meio para que ela e a filha não compartilhassem o mesmo ambiente. Recebe pensão de três salários mínimos do marido. Tem o primário completo. Relata ter lombalgia e ser portadora de artrite.

Eliana tem 44 anos, é casada e tem um filho de seis anos. Trabalha como secretária executiva bilíngüe. Apresenta renda familiar de mais de 30 salários mínimos. Realiza todas as atividades domésticas e tem uma faxineira semanal. Apresenta uma anemia importante recém-diagnosticada, em fase de averiguação.

Vera tem 41 anos, casada, com dois filhos (18 e 8 anos). Ela e o marido são autônomos e trabalham com estofamento e cortinas. A renda familiar é de aproximadamente 14 salários mínimos. Estudou até a sétima série e realiza todas as atividades da casa.

\section{Dados relacionados a demanda terapêutica e ao deficit de autocuidado}

Os dados foram analisados segundo o referencial da teoria do autocuidado, procurando-se compreender a adequação ou não da relação entre capacidade e demanda de autocuidado no adulto portador de hipertensão arterial. Nos itens em que há um desequilíbrio nesta relação, numa proporção menor de capacidade do que de demanda, podemos considerar que há déficit de autocuidado.

A demanda terapêutica de autocuidado é caracterizada a partir do levantamento dos "requisitos de autocuidado" ou "requerimentos para a ação", e é definido como tudo aquilo necessário para a regularização do funcionamento e desenvolvimento humano ${ }^{(2)}$. Estes requisitos são agrupados em três categorias universal, desenvolvimental, desvio de saúde - e geralmente guardam relação entre si.

Ainda procurou-se levantar, a partir dos fatores condicionantes, os facilitadores e dificultadores para a realização do autocuidado (Quadro 1).

No Quadro 1, evidenciamos que a demanda de autocuidado relativa ao requisito alimentação se mostrou inadequada, em alguns aspectos, nas quatro pacientes. Todas apresentam obesidade moderada segundo avaliação do índice de massa corpórea, uma delas usa dieta normossódica e outra não restringe alimentos ricos em gordura animal.

* Os nomes referidos são fictícios 
Quadro 1 - Apresentação dos requisitos de autocuidado da portadora de hipertensão arterial (HA), da demanda terapêutica de autocuidado, fatores facilitadores e dificultadores para a realização do autocuidado e o déficit de autocuidado identificado segundo a demanda terapêutica

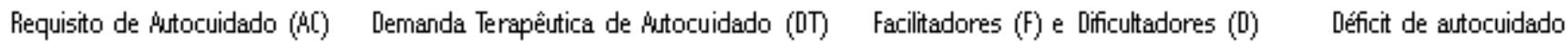

$$
\text { REQUISITOS RELACIONÁDOS AO BUTOCUIDARO UNMERSÄL }
$$

亗imentaç̃õo

Hipossódica, baixo teor de gordura saturada e colesterol; calorias necessárias para manter o Índice de Massa Corpóreaentre 19 a 28.9 - normal à obesidade leve

\section{stividade fisica}

Exercício físico regular e contínuo; sem intolerância

loana:

住学:
Faz dieta hipossódica e hipolipídica

DT: Obesidade moderada

Faz dieta hipossódica e dieta rica em E: conhece motivo da dieta e o que deve colesterol e carboidratos (é diabética)

DT: Obesidade moderada

Eliana:

Faz dieta hipossódica, hipolipídica e $\mathrm{F}$ : conhece motivo da dieta e todos Âtteração da nutrição para hipocalónica durante o dia. Come macarrão alimentos que deve evitar. Curiosa e à noite devido a falta de tempo para preparar interessada

outros alimentos

D: às vezes sente vontade de comer algo DT: Obseidade moderadae dieta inadequada fora do cardápio. Não tem conseguido perder peso, apesar da vontade

DT: Dieta com sal e gondura normal

DT: Obesidade moderada

E: não identificado

ㅁ: familiares não colaboram. Não sente necessidade de perder peso

Maria:

Caminha diariamente

loana:

Caminha duas vezes por semana; $\mathrm{F}$ : motivada para atividade física. Gosta
intolerância quando anda rápido. Realiza do contato social estabelecido com as outras atividades fisicas

Eliana:

Freqüenta academia de defesa uma vez por $\underline{F}$ : conhece beneficios dos exercícios etem semana

DT: Sedentária

Uere:

Não realiza nenhum exercício

DT: Sedentária

\section{Interação social}

Relacionamento satisfatório com familiares, vizinhos e colegas de trabalho.

Existência de amigos

\section{Maria:}

Poucos amigos, porém de qualidade 미 dificuldade em sair de casa Não há

Joana:

Ótimo relacionamento com amigos e grupo É comunicativa, gosta de fazer amizades social DT: Problemas e atritos com a filha o genro e participar de grupos

Eliana:

Sem amigos, pouco contato com a familia DT: Interação com parentes inadequada 论居:

Sem amigos ou colegas de trabaho. Parece ㅁ: marido pouco participativo e de dificil estar adaptada à situaç̃ão filha intolerante

diálogo. Procura manter seus vontade e necessidade de perder peso D: tempo restrito devido trabalho + casa + filho menor

F: já fez exercícios em academia Q : desconhece a necessidade do exerúcio. Cansa quando realiza atividades mais rápidas. Local para andar é perigoso

Inatividade fisica relacionada com a falta de tempo para realizar caminhadas ou freqüentar academia três vezes por semana

Inatividade fisica relacionada à falta de tempo, ao desconhecimento sobre a finalidade do exercício e falta de lugar adequado para andar

Déficit no relacionamento com a familia relacionado à dependência econômica da

D: mostra-se de relacionamento difícil/ Interação social prejudicada devido ao comportamento intolerante relacionamentos dentro da familia 
Lazer e recreacão

Prática de alguma forma de lazer

Prevenção de riscos

Não uso de álcool e fumo.

Riscos decorrentes da hipertensão

\section{Manutenção da saúde $\quad$ Maria:}

Procura serviço de saúde na vigência Segue orientações médicas e freqüenta de algum problema ou para chack up. regularmente o serviço de saúde Procura seguir as orientações do profissional de saúde

Promoção do desenvolvimento humano de acordo com os potenciais e limitações; satisfação com a vida

Maria:

Lazer em casa (Th, crochê). Gostaria de ir ao teatro e a concertos

loana: Eliana:

semana

Uera:

Maria:

loana: (come doce)

quanto a riscos

Eliana: se incomodada com o hábito

DT: Mantendo Pá elevada

Pera:

Pressão arterial normal

DT: Tabagista há nove anos

Joana: médicas

Eliana: com regularidade

Uera:

Faz exame ginecológico e clínico

DT: Não segue orientaç̃óes

\section{Maria:}

Intenso. Participa de vários grupos $\mathrm{E}$ : procura fazer tudo de que gosta. Não há (dança, terceira idade, ginástica) Extrovertida e comunicativa

Lazer com atividades em casa $\underline{\text { : }}$ tem carro e bom nível de Não há (bondado). Gostaria de sair aos finais de esclarecimento

Refere lazer realizando atividades ㅁ: esposo não gosta de sair e falta Não há domésticas. Não pensa sobre isto tempo durante a semana

Năo usa drogas. Sem dados paraapurar E: história de cessação do fumo

complicação da doença. Mantendo $\underline{\underline{0}}$ : idade como facilitador para as hipertensã̃o diastólica complicą̧̃oses da doença

Dieta normolipídica $\quad$ [: não consegue realizar o tratamento

DT: Não segue dieta para diabetes dietético esperado. Sente-se

DT: Hipertensão diastólica. Não aualiada diariamente

Bebia diariamente mas parou, por sentir- $\underline{F}$ : força de vontade. Conhece os riscos

da hipertensão

D: labilidade tensional com descontrole importante da PA

D: sem necessidade de parar de fumar. Desconhece riscos da doença

F: interesse sobre a saúde

[i: depende do sobrinho para levá-la ao serviço de saúde. Sobrinha doente em casa

ปT: Não segue todas as orientações F: tem tempo e disposição

ㅁ: irrita-se com a demora do atendimento. "Fico perdendo tempo aqui"

Segue orientações, procura homeopatia $\underline{F}$ : bom nível de orientação quanto à saúde. Boa disposiç̧ão para cuidar-se

D: desconhecimento importante sobre a hipertensão

Conversa sobre os problemas com a F: gosta "do novo"

irmã. Gosta de ler coisas que ampliem o $\underline{\text { Di }}$ : sente-se sobrecarregada tendo que conhecimento Duidar do marido operado do coraçãa DT: Quando está ansiosa chora, fica e da sobrinha doente calada e faz trabalho manual loana:

Átrito com a filha casada que mora com F: participa intensamente da vida. Tem ela. Participa de vários grupos sociais óbjetivos claros e procura alcançá-los. (dança, religião, terceira idade)

Tenta solucionar os problemas que a

Eliana: incomodam (filha). 价iva

Não foi identificada rede de suporte $\underline{F}$ : verbaliza a necessidade de social desenvolvimento pessoal. Tenta DT: Tensa, demonstra pouco prazer com modificar as áreas de atrito (marido, a vida e irrita-se com as coisas do dia- educação filho, chefe)

a-dia ligadas à familia e ao trabalho ㅁ: gostaria de mais tempo liure para fazercoisas ligadas aodesenvolvimento

Uera:

Ambiente familiar tranqüilo. Quando E: bom ambiente familiar. Apoio e estressada cala-se e procura andar. Na compreensão dos filhos. Sente-se religião desabafa e tem paz satisfeita Di: estagnada. Sobrecarregada com as atividades
Potencial para diminuição da perfusão em cérebro, coração e rins devido à hipertensão

Alteração da nutrição para mais doque ocorpo necessitapornão priorizar a doença e potencial para redução da perfusão em cérebro, coração e rins

Potencial para diminuição da perfusão em cérebro, coração e rins devido à hipertensão

Desconhece a doença e potencial para redução da perfusão pelo fumo

Não há

Déficit de conhecimento sobre a hipertensão

Não há

Déficit de conhecimento importante sobre a doença

Angústia espiritual

Não há

Déficit de lazer relacionado com a falta de tempo. Intolerância diante das pessoas e das dificuldades. Isolamento social 
REQUISITOS RELACIONAOOOS AOS DESUIOS DE SAIULE

Compreensão do processo saúde/ Maria:

doençą

Sem atterações

E: interesse e boa disposição para Não há

causa, gravidade, complicarções da

hipertensão. Percepção da

necessidade de tratamento

loana:
DT: itribui a doença ao nervoso e quando
acha que apressão está alta, faz repouso

Eliana:

Bom conhecimento sobre a doença

E: curiosae interessadaem aprender Não há

iera:

DT: Desconhecimento quanto a causa, 모 desinteresse

tratamento e conseqüências da doença

\section{üdesão regime médico}

Maria:

Wai às consultas e toma a DT: Interrompe drogadevido ao preço e

medicação aprender

Q: idade e limitações na marcha

D: dispersa

Déficit de conhecimento relacio-

nado à doenç̧a devido a crenças

irracionais

Déficit de conhecimento sobre a doença. Desmotivação

Potencial para aumento da pressäo arterial devido ao baixo poder econômico e falta de sistema que a ajude a usar o medicamento

loana:

DT: Interrompe droga devido ao preço e $\underline{\underline{D}}$ : senil, depende de familiares para Potencial para amento dapressão por esquecimento levá-la às consultas. Baixo poder arterial pela dificuldade econômica

Eliana: aquisitivo

DT: Interrompe droga devido ao $\underline{E}$ : às vezes ganha a medicação; sem esquecimento, apesarde deixá-los a vista efeitos colaterais

Uera:

Sem medicação prescrita, lià às consultas E: marido cobra que se trate Não há

üustamento do estilo de vida para Maria:

lidar com as mudanças de saúde Sim

loana:

Na๊o

lá comentado nos itens anteriores

Eliana:

Não

tera:

Nล๊o

REQUISITOS RELACIONAOOS AO DESENUOLUIMENTO

Controle da natalidade sem Maria:

hormônios

Menopausa

Não há

loana:

Menopausa

Não há

Eliana:

Uso de métodos naturais e de barreira

Não há

iera:

Não controla a natalidade
Potencial para aumento da pressão devido à falta de sistema que a ajude a fazer uso da droga 
Os motivos para esses déficits foram distintos nas quatro pacientes: ocorreram desinteresse em perder peso, não priorização de comportamentos que beneficiem a condição clínica, estilo de vida sedentário e falta de apoio familiar para a realização da dieta. Os motivos encontrados reforçam a necessidade de se trabalhar com referenciais de enfermagem que proporcionem condições de abordagem educativa individualizada para o tratamento nãofarmacológico.

Esses aspectos devem ser relevados, pois é sabido que o sobrepeso e o excesso de consumo de sódio podem elevar os níveis pressóricos e que a dieta saturada em gordura acelera o processo aterosclerótico na hipertensão.

0 facilitador para a realização deste requisito que apareceu em maior freqüência foi o conhecimento do motivo da dieta, e o dificultador constituiu-se na dificuldade em manter a dieta regularmente. Quanto à necessidade de perderem peso, detectamos desconhecimento sobre a relação da obesidade e hipertensão e desinteresse em emagrecerem. Com isto, inferimos haver uma dificuldade em manter certos comportamentos por toda a vida, principalmente o hábito alimentar, que envolve não só prazer, mas interação social, estilo de vida e convívio familiar.

Em relação à atividade física, observamos que duas pacientes a realizam conforme recomendação médica e duas relatam dificuldade devido a falta de tempo. Identificamos como facilitador a motivação mesmo que não relacionada à doença. 0 desconhecimento dos benefícios da atividade física é um dificultador encontrado, que, junto à outras problemáticas vivenciadas pela mulher - educação dos filhos, manutenção da casa e outros - prejudicam a realização de atividade física regular.

Quanto à interação social, das quatro investigadas somente uma mantém grupo de amigos, estabelecendo uma relação tanto de lazer, como de apoio para as situações difíceis. Vera mantém seu relacionamento mais restrito aos familiares (mãe, irmãos) e Eliana, ao marido e filho menor.

Os dificultadores encontrados constituíram-se na limitação física para sair sozinha, personalidade introvertida, preservação de hábitos de vida voltados para a família e a valorização que atribuem aos contatos sociais.

No que tange ao lazer, somente uma paciente procura e realiza atividades de recreação, considerando-as muito gratificantes. Duas pacientes gostariam de sair, mas não têm companhia e uma paciente relatou nunca ter pensado em distrair-se. Como em relação a interação social, consideramos as opções de lazer relacionadas às características da pessoa, bem como aos elementos de seu convívio.

Quanto aos comportamentos que podem favorecer as complicações da hipertensão, constatou-se que uma paciente fuma e desconhece os efeitos do fumo sobre a morbidade da hipertensão, outra interrompe 0 tratamento medicamentoso com freqüência, estando com a pressão elevada no dia da consulta médica. Joana, por ser diabética e ingerir alimentos normolipídicos, apresenta grande possibilidade de complicações cérebro e cardiovascular e também encontrava-se com a pressão arterial alterada, no dia da consulta.

A manutenção da saúde foi averiguada quando relacionavase com a hipertensão e com a fase de desenvolvimento das pacientes, caracterizada por mulheres adultas ou senis. As pacientes procuram o serviço de saúde para fazerem controle da hipertensão, e três delas relataram tentar seguir as orientações médicas. A paciente que não segue as orientações apresenta um nível de desinformação importante em relação à hipertensão, mas freqüenta com regularidade outros serviços de saúde para fazer avaliação médica periódica. Mesmo com a falta de tempo referida pelos sujeitos e a dependência de uma delas quanto ao sobrinho levá-la ao serviço de saúde, constatou-se que procuram cuidar-se.

Três pacientes verbalizaram a necessidade de maior desenvolvimento humano. Destas, uma tem mais oportunidade de fazê-lo por estar aposentada e não ter atividades em casa que a prendam. Duas encontram-se limitadas pelo excesso de atividades diárias, apesar de Maria dispor da leitura como forma de desenvolvimento.

Quanto à satisfação com a vida e os problemas vivenciados no cotidiano, apresentam dificuldades para trabalhá-los em nível mais afirmativo. As problemáticas do cotidiano geram insatisfação, stress, instabilidade da pressão arterial e desviam a atenção dos sujeitos no que diz respeito à realização de comportamentos voltados para a saúde. Esses aspectos devem ser considerados pelo enfermeiro, com o objetivo de oferecer condições ao paciente de rearranjar sua condição de vida para melhor enfrentamento dos estímulos internos e externos.

Também foi constatado que as pacientes têm uma rede de suporte social limitada, o que diminui as formas de apoio à pessoa.

A rede de apoio pode ser de fundo emocional e/ou funcional, e é definida como "a qualidade das relações que é capaz de estabelecer na própria rede social" ${ }^{(5)}$. Essas relações proporcionam sentimento de solidariedade, confiança, valorização e melhor adequação do indivíduo as situações difíceis ou problemáticas.

Quanto à compreensão do processo saúde-doença relacionado à hipertensão, encontrou-se déficit importante em duas pacientes. Uma delas só considera a elevação da pressão arterial quando na presença de sintomas físicos e outra apresenta total desconhecimento sobre a doença hipertensiva e os cuidados relativos à mesma. Essas diferentes demandas terapêuticas, pontuam deficiência de conhecimento e o desinteresse e a falta de 
motivação em questões ligadas à doença.

A adesão ao tratamento medicamentoso é inadequada em três pacientes, o que atribuem ao preço do medicamento e ao esquecimento de ingeri-lo.

Temos que pensar no significado de ser hipertenso para o indivíduo. Essa é uma doença assintomática, que só traz anormalidades ao organismo após algum tempo de instalada e quando já existe comprometimento de órgãos-alvos. Além do mais, quando há sintomas - dor de cabeça e no pescoço, nervoso, irritação, tonteira - esses são comuns a situaç̃̃es estressoras vivenciadas no dia-a-dia, e portanto, não associam a problemas com a pressão arterial $^{(6)}$.

Quanto aos requisitos relacionados ao desenvolvimento não foi detectada nenhuma anormalidade.

\section{REFERÊNCIAS BIBLIOGRÁFICAS}

1. Chompré RR. Autocuidado: necessidade ou responsabilidade? Rev Baiana Enfermagem 1994; 7(1/2):153-61.

2. Orem DE. Nursing - concepts of practice. $5^{\mathrm{a}}$ ed. St Louis: Mosby; 1995.

3. Dupas G. Reflexão e síntese acerca do modelo do autocuidado de Orem. Acta Paul Enfermagem 1994; 7(1):19-26.

4. Hartweg DL. Dorothea Orem. Self-care deficit theory. California: Sage; 1991.

\section{CONCLUSÃO}

A utilização da TDAC nos possibilitou traçar os requisitos de autocuidado necessários à pessoa hipertensa e levantar a demanda terapêutica de autocuidado. A partir daí pudemos conhecer a existência ou não de déficit de autocuidado e os aspectos que possam estar contribuindo negativa ou positivamente para a deficiência encontrada. Compreende-se que estes aspectos são indicadores importantes a serem trabalhados pelo enfermeiro ao usar a teoria do autocuidado para guiar suas ações assistenciais.

Sentimos necessidade, ao final de um estudo como este, de colocar em prática com a mesma clientela outro referencial teórico da enfermagem, e então averiguarmos a existência ou não de outras possibilidades de direcionamento das ações do enfermeiro.

5. Maciel CLC. Emoção, doença e cultura: o caso da hipertensão essencial. In: Romano BW. A prática da psicologia nos hospitais. São Paulo (SP): Pioneira; 1994.

6. Cade NV. Aspectos psicossociais no cotidiano dos hipertensos: um estudo sobre a descontinuidade do tratamento em pacientes pertencentes ao programa para controle da hipertensão arterial. [dissertação]. Vitória (ES): Universidade Federal do Espírito Santo/ UFES; 1996. 